\title{
Alteration of immunoproteome profile of Echinococcus granulosus hydatid fluid with progression of cystic echinococcosis
}

Chun-Seob Ahn ${ }^{1 \dagger}$, Xiumin Han ${ }^{2 \dagger}$, Young-An Bae ${ }^{3}$, Xiao Ma ${ }^{2}$, Jin-Taek Kim ${ }^{1}$ Huixia Cai ${ }^{1,2}$, Hyun-Jong Yang ${ }^{4}$, Insug Kang ${ }^{5}$, Hu Wang ${ }^{2}$ and Yoon Kong ${ }^{1 *}$

\begin{abstract}
Background: Cystic echinococcosis (CE), caused by Echinococcus granulosus metacestode, invokes a serious public health concern. Early diagnosis has great impacts on reduction of disability-adjusted life years. Several antigen B-related molecules (EgAgB; EgAgB1-5) are known to be immunopotent, but detection of EgAgB is variable in many patients and may not allow reliable interpretation of its immunological relevance. More importantly, the immunoproteome profile of hydatid fluid (HF) has not been addressed.

Methods: We conducted a proteome analysis of the HF of a single fertile cyst of CE1 and CE2 stages through two-dimensional electrophoresis (2-DE). Each protein spot was analyzed by matrix-assisted laser desorption/ ionization time-of-flight mass spectrometry (MALDI-TOF-MS). We subsequently determined the immunoproteome profile employing patient sera of entire disease spectrum from CE1 to CE5 stages.

Results: We identified 40 parasite proteins, of which EgAgB (28 spots) and antigen 5 (EgAg5; 5 molecules) were abundant. EgAgB proteoforms constituted the majority, mostly EgAgB1 (24 spots), followed by EgAgB2 and EgAgB4 (2 spots each). EgAgB3 was detected only by liquid chromatography-MS/MS. EgAgB5 was not recognized. We also detected 38 host proteins, which were largely composed of serum components, antioxidant/xenobiotic enzymes, and enzymes involved in carbohydrate metabolism. CE1 and CE2 HF exhibited comparable spotting patterns, but CE2 HF harbored greater amounts of EgAgB and EgAg5 complexes. CE sera demonstrated complicated immune recognition patterns according to the disease progression; CE2 and CE3 stages exhibited strong antibody responses against diverse EgAgB and EgAg5 proteoforms, while CE1, CE4, and CE5 stages mainly reacted to EgAg5 and cathepsin B. Patient sera of alveolar echinococcosis (AE) cross-reacted with diverse EgAgB isoforms (36\%). EgAg5 and cathepsin B also demonstrated cross-reactions with sera from neurocysticercosis and sparganosis.

Conclusions: Our results demonstrated that detection of a single defined molecule may not properly diagnose CE, since specific immunodominant epitopes changed as the disease progresses. Immunoproteome analysis combined with imaging studies may be practical in the differential diagnosis of CE from AE and other cystic lesions, as well as for staging $C E$, which are pertinent to establish appropriate patient management.
\end{abstract}

Keywords: Echinococcus granulosus, Cystic echinococcosis, Hydatid fluid, Immunoproteome, Antigen B, Antigen 5, Stage-specificity

\footnotetext{
*Correspondence: kongy@skku.edu

${ }^{\dagger}$ Equal contributors

'Department of Molecular Parasitology, Sungkyunkwan University School of Medicine and Center for Molecular Medicine, Samsung Biomedical Research Institute, Suwon 440-746, Korea

Full list of author information is available at the end of the article
} 


\section{Background}

Cystic echinococcosis (CE), caused by Echinococcus granulosus metacestode, is one of the most deleterious helminthic diseases of humans and livestock. CE is detected worldwide, but it is more prevalent in the nomadic areas of Central and Middle Asia, Eastern Europe, Africa, Australia, South America, and northwestern China [1-3]. Approximately 4 million people are infected and another 40 million are at risk of infection annually [4]. Humans are infected by incidental contract with the eggs in association with dog rearing environments. Oncospheres hatched from the eggs are activated in the small intestine and subsequently penetrate the intestinal wall to enter the circulation. They mostly egress in the liver and lung, and grow slowly to hydatid fluid (HF)-filled cyst, in which many protoscoleces and daughter cysts develop [5].

Clinical manifestations of $\mathrm{CE}$ are rarely present until large cyst(s) crowd the affected organs/tissues. Approximately $60-88 \%$ of patients with cysts less than $7.5 \mathrm{~cm}$ in diameter manifest no discernible symptoms [6,7]. Most $\mathrm{CE}$ cases are diagnosed ages between the third and fifth decades, but the highest morbidity has been observed in young patients under the first two decades [8]. Therefore, early detection significantly reduces morbidity and mortality associated with $\mathrm{CE}$, which remains with challenging issues. The diagnosis of $\mathrm{CE}$ largely depends on imaging scans and serological tests. However, imaging diagnosis modalities, such as ultrasonography (US), computed tomography (CT), and magnetic resonance imaging (MRI), necessitate differential diagnosis from alveolar echinococcosis (AE), cystic lesions, liver cirrhosis, and primary hepatocellular carcinoma [5,9]. Staging of CE is also ambiguous in many cases. In such complex situations, serodiagnosis provides additional evidence for $\mathrm{CE}$ in patients with hepatic/pulmonary lesions and is beneficial to screen and diagnose patients in endemic areas [10].

Several component proteins of HF, such as diverse isoforms of antigen 5 (EgAg5) and antigen B (EgAgB) are known to be potent diagnostic antigens, among which native and recombinant EgAgBs are reliable and reproducible antigens that allow for better diagnostic performance $[5,11,12]$. EgAgB is a thermostable, macromolecular multifunctional lipoprotein encoded by a multigene family [13]. At least 10 genes in five subclasses are differentially expressed from the parasite [14]. Its functions include elastase inhibition, augmentation of $\mathrm{TH} 2$-biased immune responses, and sequestration of hydrophobic substances [11,15-17]. However, it has not been critically determined whether specific antibodies against EgAgB are detected in both the active and chronic stages of the disease or whether EgAgB shows species-specificity. Demonstration of specific antibodies against EgAgB varies from patient to patient, which does not allow clinical interpretation of its immunological significance $[5,11,16,18,19]$. More importantly, aspects of the immunoproteome profile, such as global antibody responses and alteration of specific antigens in relation to disease characteristics have not been investigated.

In the present study, we analyzed the proteome profile of a single fertile cyst of CE1 and CE2 stages through matrix-assisted laser desorption/ionization time-of-flight mass spectrometry (MALDI-TOF-MS), and identified 40 parasite proteins, in which EgAgB (28 spots) and EgAg5 (5 molecules) were abundant. We subsequently determined their antigenic properties along with $\mathrm{CE}$ progression, as well as their cross-reactions with sera of AE and other diseases. Our results demonstrate that immune recognition patterns change with disease characteristics, and further suggest that simultaneous monitoring of the immunoproteome profile and imaging scans might be required to properly diagnose and appropriately manage CE patients.

\section{Methods}

\section{Parasite samples}

Hepatic CE cysts were obtained from the naturally infected sheep slaughtered in Xining, Qinghai Province, China. Individual cysts were examined by US, and fertile CE1 and CE2 cysts were independently collected. After careful decortication, the cyst surface was washed with physiological saline more than 10 times at $4^{\circ} \mathrm{C}$. Each HF was aseptically drawn from intact cysts in the presence of a protease inhibitor cocktail (one tablet/25 ml, Roche, Germany) and centrifuged at $20000 \times \mathrm{g}$ for $1 \mathrm{~h}$ at $4^{\circ} \mathrm{C}$. Supernatants were concentrated by lyophilization and stored at $-80^{\circ} \mathrm{C}$ until use.

\section{Study subject and serum samples}

Sera of 59 hepatic CE patients (37.1\% male), whose disease statues were defined by US [3], were studied. Patients of CE1 stage $(n=11)$ showed unilocular hypodense cyst(s) with a double line sign. CE2 patients $(n=12)$ presented with multivesicular, multiseptated cysts with detached endocysts. Patients of CE3 $(n=12)$ demonstrated unilocular cysts with daughter cysts that contained a solid cyst matrix or detached endocyst. CE4 patients $(n=13)$ showed typical canalicular structure (ball of wool appearance). CE5 patients $(n=11)$ presented with heavily degenerative cyst (s) with calcified wall. Involvement of the right lobe was frequent $(71 \%)$. Eleven patients $(18.6 \%)$ had multiple cysts. Most patients (85\%) did not complain of specific symptoms/signs. Their demo-epidemiological profile, cyst locations, and representative US findings are summarized in Additional file 1 . In addition, this study included 10 sera per each of the following groups: neurocysticercosis, $\mathrm{AE}$, sparganosis, pathology-proven primary hepatocellular carcinoma, and healthy controls, all of whom denied any possible exposure to protozoan and helminthic infections $[5,20]$. Study protocol was approved by the Institutional Review Committee of Qinghai Province Institute for 
Endemic Diseases Prevention and Control (protocol no. 2013-7-22). The study was presented to the local health authorities, community leaders, and patients to ensure their acceptance and collaboration. Written informed consent was obtained from all patients. In the case of illiterate patients, consent was obtained by verbal notification.

Two-dimensional electrophoresis (2-DE) and immunoblotting Each HF $(200 \mu \mathrm{g})$ mixed with rehydration buffer $(6 \mathrm{M}$ urea, $2 \mathrm{M}$ thiourea, 2\% CHAPS, $0.4 \%$ dithiothreitol [DTT], $0.5 \%$ immobilized $\mathrm{pH}$ gradient [IPG] buffer and $0.002 \%$ bromophenol blue [BPB]), was cup-loaded on IPG strips (pH 6-11, 13 cm; GE Healthcare, Piscataway, NJ, USA), focused for a total of $25 \mathrm{kVh}$, processed with 15\% SDSPAGE $(160 \times 160 \times 1.5 \mathrm{~mm})$, and stained with Coomassie Brilliant Blue G-250 (CBB). For immunoblotting, proteins separated by 2-DE were electroblotted to nitrocellulose membranes (Santa Cruz, Dallas, TX, USA) for $8 \mathrm{~h}$ at $4^{\circ} \mathrm{C}$. The membranes were blocked with Tris-buffered saline (100 mM, pH 8.0) containing 3\% skim milk and 0.1\% Tween-20 for $1 \mathrm{~h}$. The blots were incubated with individual and/or pooled serum overnight (1:1000-dilution) at $4^{\circ} \mathrm{C}$ and sequentially with horse-radish peroxidase (HRP)-conjugated anti-human IgG (Cappel, Solon, OH, USA) for $2 \mathrm{~h}$ (1:4000-dilution). For quantitative analysis, all signals were detected with SuperSignal Chemiluminescence (ECL; GE Healthcare) after a 2 min exposure. Images were digitalized with an Umax Image Scanner (Dallas, TX, USA) and analyzed employing Progenesis software (Nonlinear Dynamics, Newcastle, UK). The individual spots, whether stained or immunoblotted, were quantified by calculation of the spot volume using the total spot volume normalization method, multiplied by the total area of all the spots.

\section{Matrix-assisted laser desorption/ionization time-of-flight mass spectrometry (MALDI-TOF-MS) and nano-liquid chromatography-electrospray ionization/multi-stage MS (nano-LC-ESI-MS/MS)}

Two different sets of in-gel trypsin-digested protein spots from each CE1 and CE2 HF were independently applied using the AB SCIEX TOF/TOF 4800 Plus System (AB SCIEX, Framingham, MA, USA). Internal standards were tryptic autodigestion peaks $(m / z=842.5099$ and 2211.1046). Monoisotopic peptide masses were selected between 600 and $3500 \mathrm{Da}$. The proteins were identified by peptide mass fingerprint (PMF) with a Mascot server. Mass tolerance was $\pm 50 \mathrm{ppm}$. One missed cleavage site was allowed. Identification was accepted when PMF harbored at least two identified peptides ( $>99 \%$ probability).

For nano-LC-ESI-MS/MS, CE1 and CE2 HF were separated by $15 \%$ reducing SDS-PAGE and visualized with $\mathrm{CBB}$ staining. Each band corresponding to $24 \mathrm{kDa}$ was cut and sliced into fragments from independent gels. Disulfide bonds were reduced with $10 \mathrm{mM}$ DTT. After alkylation, gels were dehydrated, trypsin-digested, and dried in a vacuum evaporator (MIVAC DUO, Genevac, Ipswich, UK). Analysis was conducted employing a model 1200 nano-flow system (Agilent Technologies, Palo Alto, CA, USA) connected to a LTQ linear ion trap mass spectrometer (Thermo Electron, San Jose, CA, USA). The reversed phase capillary column was $12 \mathrm{~cm}$ in length, $75 \mathrm{~mm}$ in inner diameter, and in-house packed with $5 \mu \mathrm{m}$ $200 \AA$ Å pore-sized Magic C18AQ beads (Michrome BioResources, Auburn, CA, USA). The peptides were eluted in a linear gradient from 10 to $40 \%$ acetonitrile over $65 \mathrm{~min}$. MS survey was scanned for $300-2000 \mathrm{~m} / z$ with three data-dependent MS/MS scans of isolation width $(1.5 \mathrm{~m} / z)$, normalized collision energy (25\%), and dynamic exclusion duration $(180 \mathrm{sec})$. MS data was generated in RAW file format (Thermo Scientific) using the Xcalibur 1.4 with Tune 1.0. Peptide peaks were introduced into MS/MS ions search within a Mascot server (http:// www.matrixscience.com). Mass values were selected using monoisotopic masses. Peptide and MS/MS tolerances were \pm 1.2 and $\pm 0.6 \mathrm{Da}$. Protein identifications of individual ions scores $>43$ were considered as significant or to demonstrate extensive homology $(p<0.05)$.

Cysteine carbamidomethylation and methionine oxidation were considered during the analyses. MS database search was performed on the NCBI database (http://www. ncbi.nlm.nih.gov). Signal peptide sequences were predicted by SignalP 4.1 (http://www.cbs.dtu.dk/services/SignalP/) and PSORT (http://psort.hgc.jp/form.html).

\section{Results}

Proteomic analysis of E. granulosus HF collected from different CE stages reveals similar spotting patterns, but CE2 HF harbored enriched EgAgB and EgAg5 proteoforms In order to comparatively delineate HF proteins of different $\mathrm{CE}$ stages, we determined the protein profile of a single fertile CE1 and CE2 cyst. As shown in Figure 1A and $\mathrm{B}$, equivalent spotting patterns were evident, but CE2 HF harbored more EgAgB than CE1 HF. The expression pattern of EgAg5 was also shown to be upregulated in CE2 HF (see also Table 1). We identified 78 protein spots $(p<0.01) ; 40$ Echinococcus and 38 host proteins. All the Echinococcus proteins were concomitantly identified; they typically segregated into diverse isoforms of EgAgB and EgAg5. EgAgB proteoforms appeared to be encoded by six different genes (ACZ51458, ACZ51459, and ACP21247 for EgAgB1; AAP83174 and AAW78457 for EgAgB2; and ACZ51452 for EgAgB4). Among the EgAgB complex, EgAgB1 was the major components comprising 24 molecules between 8 and $16 \mathrm{kDa}$ (spots 43-49, 60-74, 77, and 78). Each of two EgAgB4- (spots 58 and 59) and EgAgB2related proteins (spots 75 and 76) was recognized. EgAgB3and EgAgB5-related molecules could not be identified. This result matched well with previous studies in which $E g A g B 1$, 


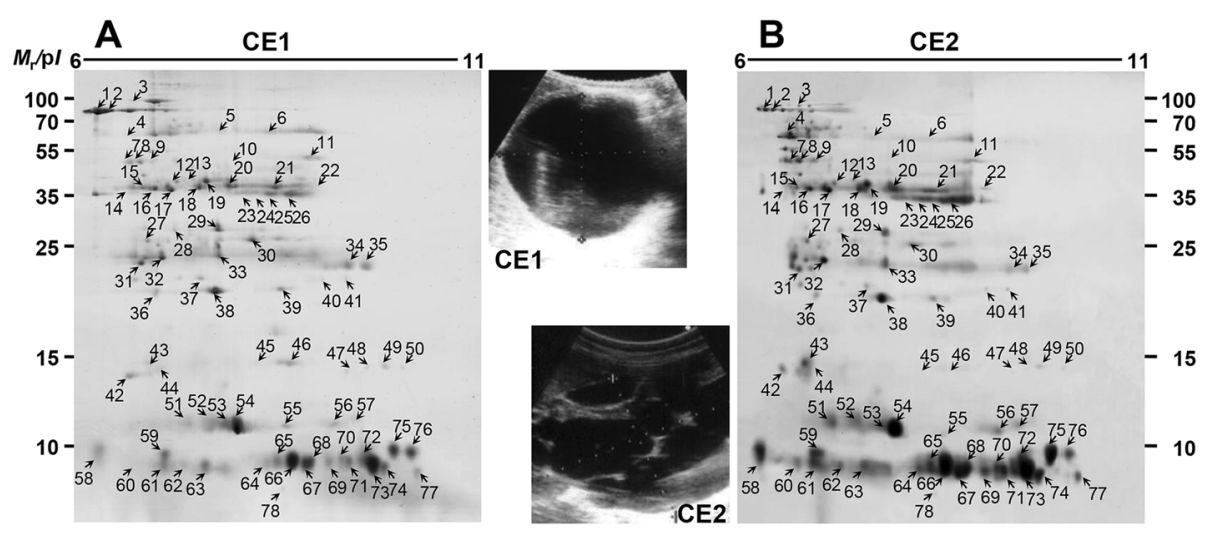

Figure 1 Electrophoretic profile of E. granulosus HF collected from sheep livers. HF (200 $\mu$ g proteins) harvested from single fertile cyst of CE1 (A) and CE2 (B) stages were electrofocused on IPG strips ( $\mathrm{pH}$ 6-11) and resolved by 15\% SDS-PAGE. The gels were stained with CBB. The protein spots were subjected to in-gel trypsin digestion and processed with MALDI-TOF-MS. Proteins identified are marked by Arabic numbers (1-78) and are summarized in Table 1 and Additional file 2. Representative ultrasonographic images of CE1 and CE2 cysts are shown. $M_{r}$, molecular weights in $\mathrm{kDa}$; $\mathrm{pl}$, isoelectric point.

$E g A g B 2$, and $E g A g B 4$ were abundantly transcribed and expressed among several EgAgB isoforms [14,21]. Five EgAg5 isoforms/variants were detected (spots 21 and 23-26; ADG65665). Other molecules (7 spots) included enzymes involved in carbohydrate metabolism (5 molecules), such as phosphoenolpyruvate carboxykinase (spot 2), citrate synthase (spot 10), phosphoglycerate kinase (spot 11 ), fructose 1,6 biphosphate aldolase (spot 22), and triophosphate isomerase (spot 32), and proteolytic cathepsin B (2 molecules; spots 7 and 9) (Table 1 and Additional file 2).

We also identified 38 host-derived ovine proteins. These molecules mainly consisted of serum components (serotransferrin, carbonic anhydrase, and immunoglobulin and hemoglobin families). In addition, antioxidant/detoxification/xenobiotic proteins (selenium-binding protein 1 , dihydrodiol dehydrogenase 3 , peroxiredoxin-1, and glutathione transferases), enzymes involved in carbohydrate (sorbitol dehydrogenase and alcohol dehydrogenase) and amino acid metabolism (fumarylacetoacetase), fatty-acidbinding proteins (lipid transporter), phosphatidylethanolamine-binding protein (signaling molecule), oxidoreductase (glycerol-3-phosphate dehydrogenase and carbonyl reductase), sugar-binding galectin-3, and $\gamma$-tubulin were detected (Additional file 2).

Immune recognition profile of HF against sera of different CE stages are altered along with CE progression and differed from one another

The EgAgB complex has been well known to induce specific antibody responses against $\mathrm{CE}$ sera $[1,5,10,11,16]$,

Table 1 Identification of E. granulosus proteins in CE1 and CE2 cysts by MALDI-TOF-MS

\begin{tabular}{llll}
\hline Spot no(s). & Theoretical $\boldsymbol{M}_{\mathbf{r}} / \mathbf{p} \boldsymbol{I}$ & Accession no & Description $^{\mathbf{a}^{2}}$ \\
\hline 2 & $55590 / 8.39$ & CBH36490 & Phosphoenolpyruvate carboxykinase \\
7,9 & $39841 / 6.82$ & EgrG_000790200 & Cathepsin B \\
10 & $51345 / 8.26$ & EgrG_001028500 & Citrate synthase \\
11 & $44227 / 8.03$ & EUB59398 & Phosphoglycerate kinase \\
$21,23-26$ & $33316 / 9.02$ & ADG65665 & 38 kDa antigen 5 \\
22 & $39666 / 8.31$ & EgrG_000905600 & Fructose 1,6 bisphosphate aldolase \\
32 & $27123 / 6.60$ & EgrG_000416400 & Triosephosphate isomerase \\
$43-49,60-71,74,77,78$, & $6232 / 8.31$ & ACZ51459 & EgAgB1/1 \\
72 & $5348 / 9.30$ & ACP21247 & EgAgB1 subunit, partial \\
73 & $7223 / 9.23$ & ACZ51458 & EgAgB1 \\
58,59 & $8337 / 6.78$ & ACZ51452 & EgAgB4/1 \\
75 & $9282 / 9.38$ & AAW78457 & EgAgB2 \\
76 & $9314 / 9.38$ & AAP83174 & EgAgB subunit 2 \\
\hline
\end{tabular}

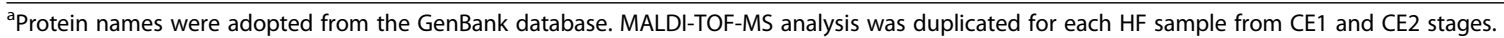

${ }^{\mathrm{b}}$ The presence of a signal peptide (SP) sequence was predicted by SignalP 4.1 and PSORT. 
while analysis of the global immunoproteome profile and changes of specific epitopes along with CE characteristics have not been explored [19]. In order to establish a global immunoproteome profile, we observed the immunorecognition pattern of HFs of sheep CE1 and CE2 cysts by using pooled patient serum from CE1-CE5 stages $(n=3$ per group). These HFs demonstrated an immunoreactivity profile similar to each other, but CE2 HF exhibited more distinct patterns than CE1 (left panel, Figure 2A; other data not shown). We used CE2 HF to compile the CE immunoproteome.

When blots containing CE2 HF were probed with a pooled CE patient serum, cathepsin B (spots 7 and 9), EgAg5 (spots 23-26), and diverse EgAgB proteoforms demonstrated strong antibody reactions in a highly complicated fashion. Interestingly, five to seven spots located at approximately $24 \mathrm{kDa}$, which could not be identified through MALDI-TOF-MS analysis, displayed positive reactions (box, Figure 2A). We analyzed the corresponding band (right panel, Figure 2A) by LC-ESI-MS/MS and recognized that this band contained EgAgB1, EgAgB2, EgAgB3, and EgAg4 isoforms (Figure 2B).

Among the diverse EgAgB proteoforms, EgAgB1 (spots 44, 61-69, 71, 73, 74, and 77) revealed high antibody capturing activity, which comprised $55.1 \%$ of the total volume of the proteins and $80.2 \%$ of the total volume of the antigenic spots (dotted-boxes, Figure 2C). Other EgAgB subunits, such as EgAgB2, EgAgB3, and EgAgB4 exhibited minimal antibody reactivity. This result convincingly demonstrated that $\mathrm{EgAgB1}$ isoforms constituted immunodominant antigens.

We subsequently determined that the alteration of immunoreactive spots in accordance with CE involution (Figure 3). When we probed individual CE1 sera, immunoreactions against EgAg5 (spots 23-26) and cathepsin B (spot 9) were fairly high (36.4-72.7\%). Conversely, EgAgB1 isoforms, such as spots 47, 48, 61, 63, 66, 67, $69,72-74$, and 77 showed relatively weak responses

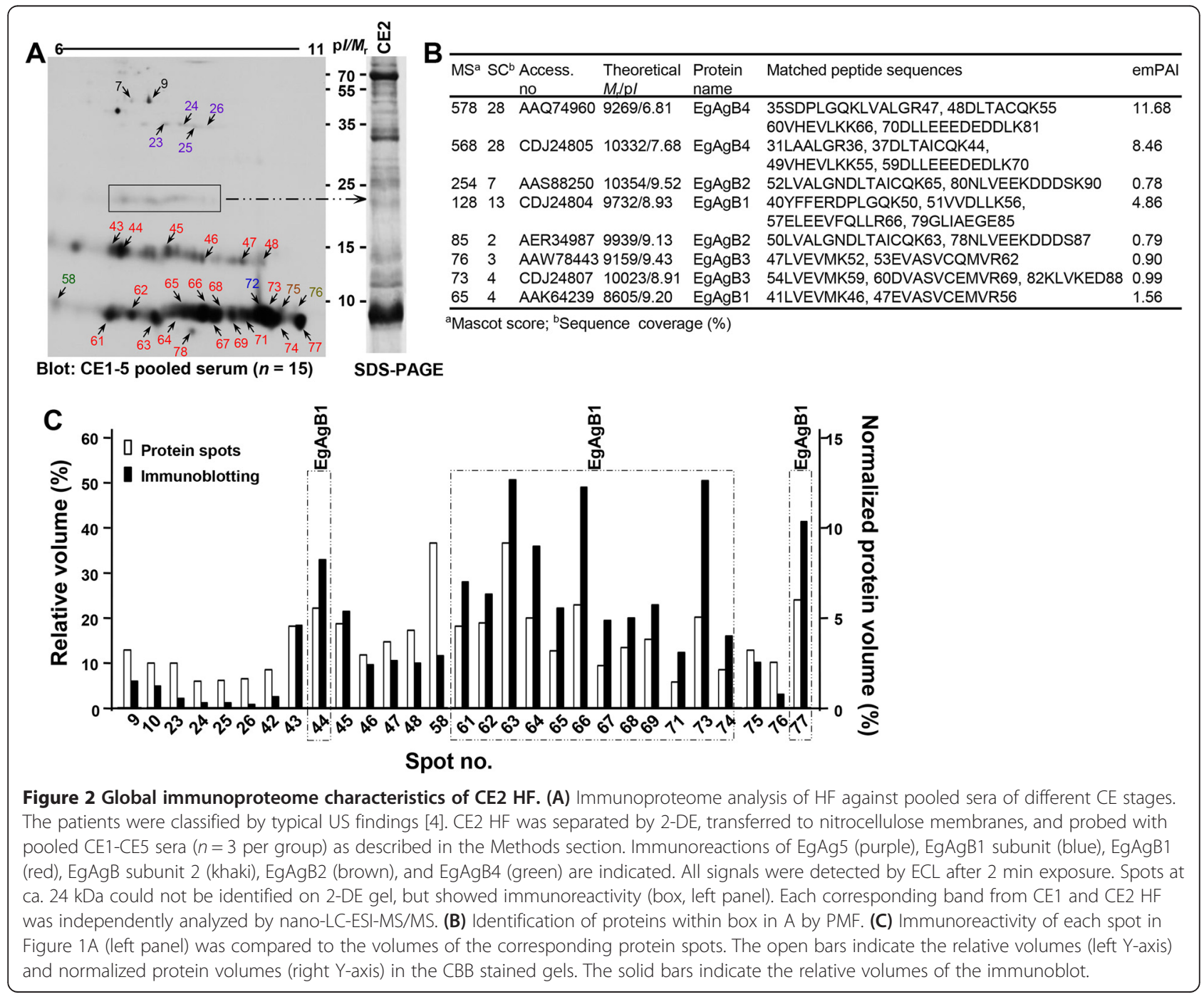




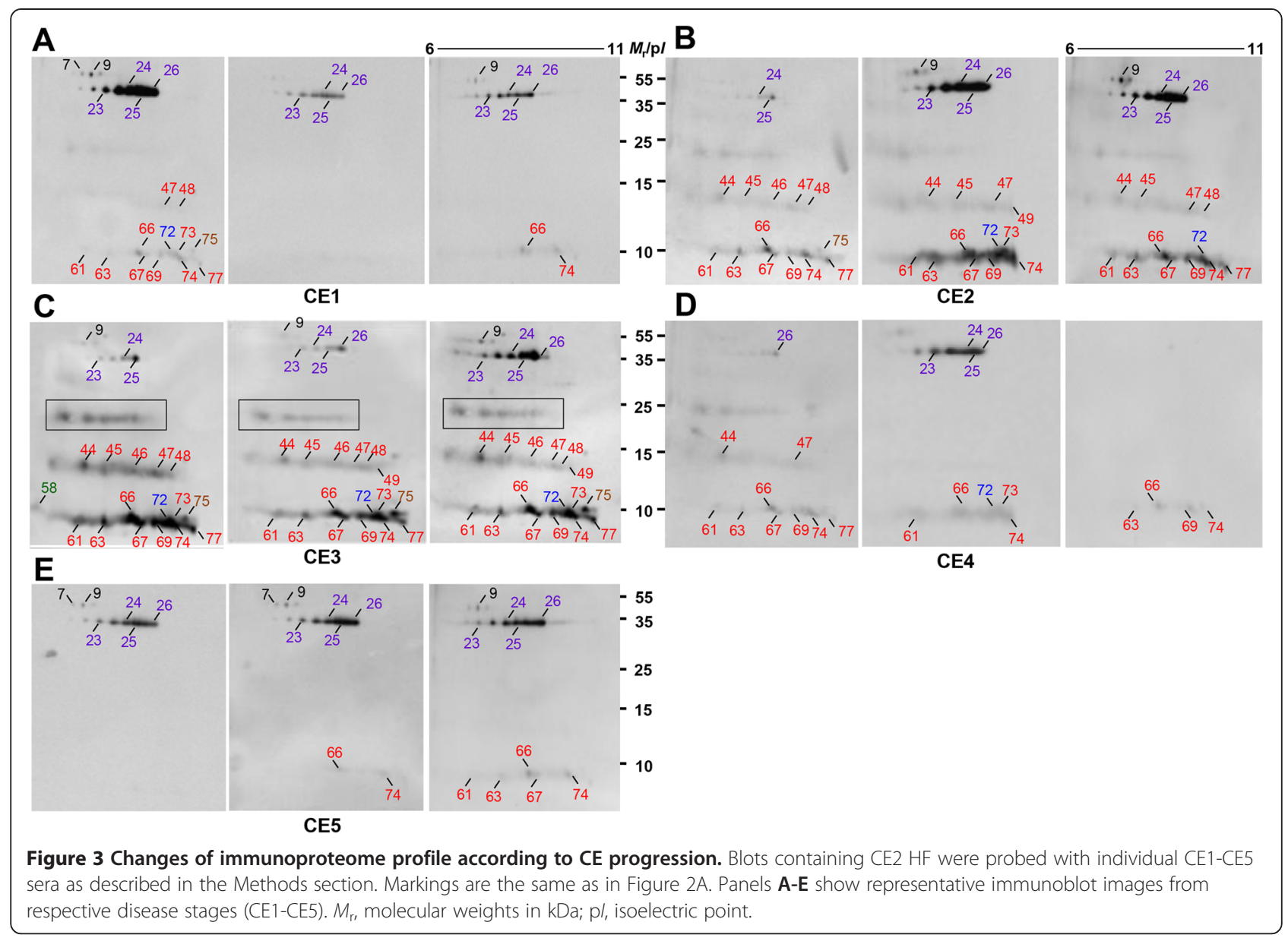

(9.1-54.5\%) (panel A). CE2 patient sera demonstrated dissimilar immune recognition patterns as compared to those of CE1; reactions against EgAgB subunits, especially against $8 \mathrm{kDa}$ isoforms became evident with growing frequency $(75-100 \%)$, with the exception of spot 61 (33.3\%). Cathepsin B (spot 9) and EgAg5 (spots 23-26) also showed positive responses (75-91.7\%) (panel B). As the disease progressed to the transitional CE3 stage, a variety of EgAgB isoforms (spots 44-48, $61,63,66,67,69,72-75$, and 77) exhibited prominent antibody responses (75-100\%). EgAg5 (spots 23-26) also revealed positive reactions. In this stage, five to seven spots located at approximately $24 \mathrm{kDa}$, which were identified as various EgAgB isoforms (Figure 2B), demonstrated positive reactions as well (panel C). As the disease progressed to inactive CE4 and CE5 stages, the immunoproteome profile regressed toward that observed in CE1. Cathepsin B (spot 9) and 38 kDa EgAg5 subunits (spots 23-26) retained their responsiveness (30.8-85.7\%), while EgAgB proteoforms revealed significantly decreased antibody capturing activity $(0-63.6 \%)$ (panels D and E).

\section{Assessment of cross-reactivity}

We determined whether or not the proteins that showed high immunoreactivity against $\mathrm{CE}$ sera cross-reacted with heterologous infection sera and hepatocellular carcinoma patient sera. When pooled serum of AE patients $(n=10)$ was probed with CE2 HF, cathepsin B (spots 7 and 9), EgAg5 (spots 23-26), EgAgB2 (spot 75), and some EgAgB1 isoforms (spots 44-48, 61-64, 66-69, 71-74, and 77) exhibited serological cross-reactions. Further analyses employing individual $\mathrm{AE}$ sera demonstrated cross-reactivity of EgAg5 (70-80\%) and EgAgB1 (20-50\%) with a complicated pattern (Figure 4A). Pooled serum (each of $n=10$ ) or individual sera of neurocysticercosis and sparganosis also exhibited weak cross-reactions with certain isoforms of EgAg5 (spots 23-26) and EgAgB (spots 66, 73, and 75) (Figure 4B and C). Serological cross-reactions between $\mathrm{CE}$ and $\mathrm{AE}$ may be inherent to some extent due to the highly conserved sequence identity between EgAgBs and EmAgBs (78.8-94.2\%), except for the antigen B3 series (36.5\%) (data not shown), which did not invoke a significant antibody response against $\mathrm{CE}$ patients. Primary hepatocellular carcinoma patient sera and sera of 


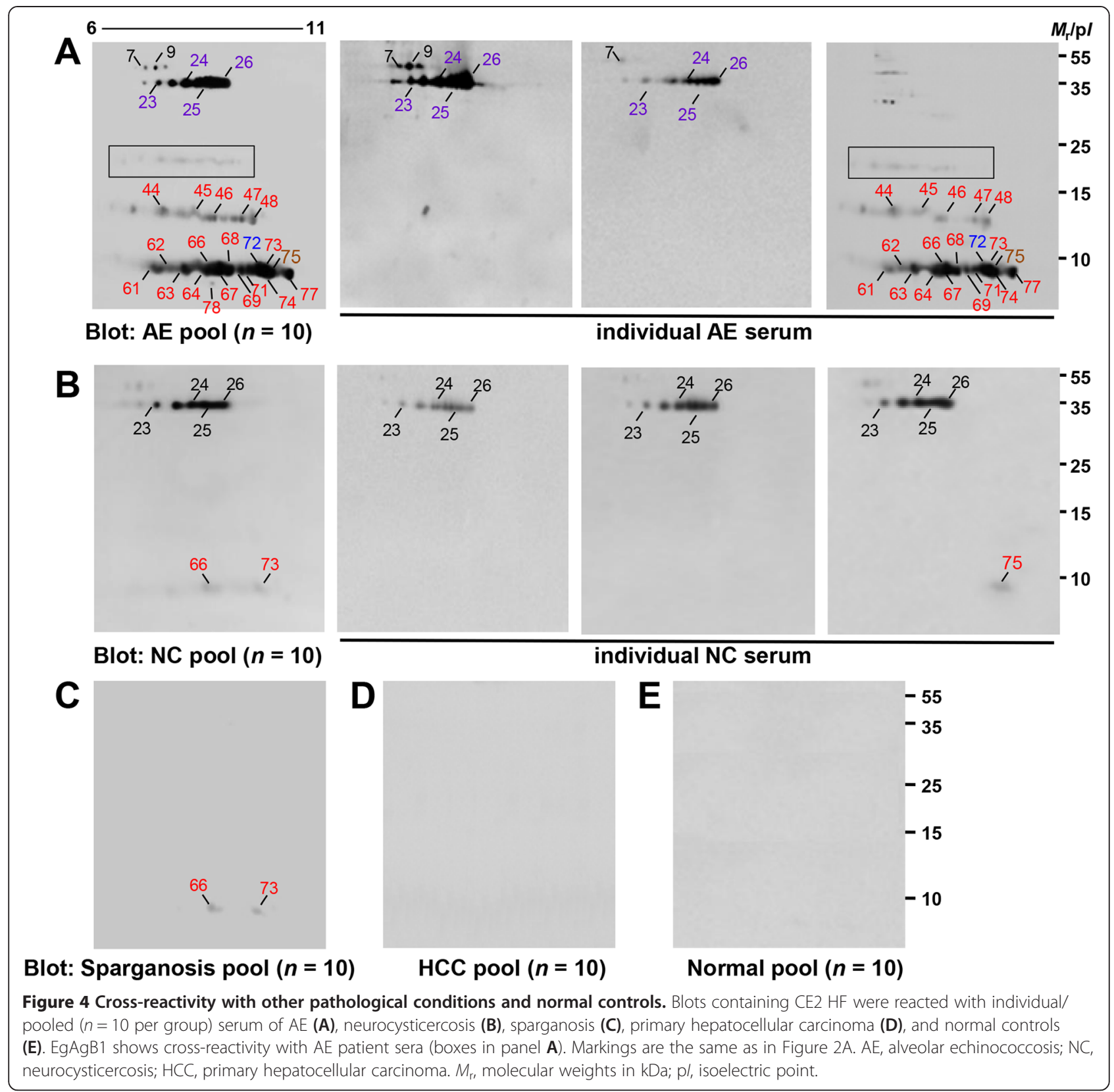

normal controls did not show any detectable crossreactivity (Figure 4D and E). Table 2 summarizes the evaluation of sensitivity and specificity of important diagnostic spots by $2-\mathrm{DE} /$ immunoblotting.

\section{Discussion}

In this study, we have undertaken a comprehensive proteome analysis of CE1 and CE2 HF and identified 78 individual protein spots (40 Echinococcus and 38 host proteins), in which EgAgB (28 species) and EgAg5 (5 molecules) were most abundantly recognized. Among the EgAgB series, EgAgB1 represented the most profoundly expressed proteoforms regardless of $\mathrm{CE}$ status, which was consistent with previous proteomic analysis of ovine CE HF [21]. We subsequently determined the antigenic properties of the respective proteoforms along with $\mathrm{CE}$ progression and established a global CE immunoproteome profile for the first time.

Our results demonstrated that immune recognition patterns of $\mathrm{CE}$ patients were altered along with the disease progression and they differed from one another. In both the early $\mathrm{CE} 1$, and the chronic inactive CE4 and CE5 stages, EgAg5 played a major role in inducing antibody responses. This result suggests that posttranslationally modified, externally exposed phosphorylcholine moieties of the EgAg5 (a major antigenic epitope), 
Table 2 Responsiveness of major antigenic spots of E. granulosus HF

\begin{tabular}{|c|c|c|c|c|c|c|c|c|c|c|}
\hline \multirow{2}{*}{$\begin{array}{l}\text { Spot no. } \\
\text { (Description }^{\mathrm{a}} \text { ) }\end{array}$} & \multicolumn{6}{|c|}{ No. of positive (\% sensitivity) } & \multicolumn{4}{|c|}{ No. of negative (\% specificity) } \\
\hline & $\begin{array}{l}\text { CE1 } \\
(n=11)\end{array}$ & $\begin{array}{l}\text { CE2 } \\
(n=12)\end{array}$ & $\begin{array}{l}\text { CE3 } \\
(n=12)\end{array}$ & $\begin{array}{l}\text { CE4 } \\
(n=13)\end{array}$ & $\begin{array}{l}\text { CE5 } \\
(n=11)\end{array}$ & $\begin{array}{l}\text { Overall } \\
(n=59)\end{array}$ & $\begin{array}{l}\mathrm{AE} \\
(n=10)\end{array}$ & $\begin{array}{l}\mathrm{NC} \\
(n=10)\end{array}$ & $\begin{array}{l}\text { Others }^{\mathrm{b}} \\
(n=30)\end{array}$ & $\begin{array}{l}\text { Overall } \\
(n=50)\end{array}$ \\
\hline 9 (Cathepsin B) & $5(45.5)$ & $9(75)$ & $12(100)$ & $4(30.8)$ & $6(85.7)$ & $36(61)$ & $3(30)$ & $9(90)$ & $30(100)$ & $42(84)$ \\
\hline 23 (38 kDa EgAg5) & $4(36.4)$ & $11(91.7)$ & $12(100)$ & $7(53.9)$ & $9(81.8)$ & $43(72.9)$ & $2(20)$ & $6(60)$ & $30(100)$ & $38(76)$ \\
\hline 24 (38 kDa EgAg5) & $5(45.5)$ & $10(83.3)$ & $12(100)$ & $8(61.5)$ & $8(72.7)$ & $43(72.9)$ & $2(20)$ & $6(60)$ & $30(100)$ & $38(76)$ \\
\hline 25 (38 kDa EgAg5) & $6(54.5)$ & $10(83.3)$ & $12(100)$ & $8(61.5)$ & $8(72.7)$ & $44(74.6)$ & $2(20)$ & $6(60)$ & $30(100)$ & $38(76)$ \\
\hline 26 (38 kDa EgAg5) & $8(72.7)$ & $10(83.3)$ & $12(100)$ & $7(53.8)$ & $9(81.8)$ & $46(80.0)$ & $3(30)$ & $6(60)$ & $30(100)$ & $39(78)$ \\
\hline $44(\mathrm{EgAgB1} / 1)$ & $0(0)$ & $10(83.3)$ & $10(83.3)$ & $7(53.8)$ & $0(0)$ & $27(45.8)$ & $8(80)$ & $10(100)$ & $30(100)$ & $48(96)$ \\
\hline $45(\mathrm{EgAgB1} / 1)$ & $0(0)$ & $9(75)$ & $10(83.3)$ & $6(46.2)$ & $0(0)$ & $25(42.4)$ & $7(70)$ & $10(100)$ & $30(100)$ & $47(94)$ \\
\hline $46(\mathrm{EgAgB1} / 1)$ & $0(0)$ & $10(83.3)$ & $11(91.7)$ & $5(38.5)$ & $0(0)$ & $26(44.1)$ & $8(80)$ & $10(100)$ & $30(100)$ & $48(96)$ \\
\hline 47 (EgAgB1/1) & $4(36.4)$ & $10(83.3)$ & $10(83.3)$ & $6(46.2)$ & $0(0)$ & $30(50.8)$ & $8(80)$ & $10(100)$ & $30(100)$ & $48(96)$ \\
\hline 48 (EgAgB1/1) & $3(27.3)$ & $9(75)$ & $10(83.3)$ & $5(38.5)$ & $0(0)$ & $27(45.8)$ & $7(70)$ & $10(100)$ & $30(100)$ & $47(94)$ \\
\hline 61 (EgAgB1/1) & $1(9.1)$ & $4(33.3)$ & $9(75)$ & $5(38.5)$ & $3(27.3)$ & $22(37.3)$ & $6(60)$ & $10(100)$ & $30(100)$ & $46(92)$ \\
\hline 63 (EgAgB1/1) & $4(36.4)$ & $9(75)$ & $12(100)$ & $7(53.9)$ & $6(54.5)$ & $38(64.4)$ & $7(70)$ & $10(100)$ & $30(100)$ & $47(94)$ \\
\hline $66(\mathrm{EgAgB1} / 1)$ & $6(54.5)$ & $10(83.3)$ & $12(100)$ & 11 (84.6) & $5(45.5)$ & $44(74.6)$ & $5(50)$ & $8(80)$ & $28(93.3)$ & $41(82)$ \\
\hline 67 (EgAgB1/1) & $5(45.5)$ & $9(75)$ & $12(100)$ & $5(38.5)$ & $2(18.2)$ & $33(55.9)$ & $7(70)$ & $10(100)$ & $30(100)$ & $47(94)$ \\
\hline 69 (EgAgB1/1) & $5(45.5)$ & $9(75)$ & $12(100)$ & $7(53.8)$ & $0(0)$ & $33(55.9)$ & $6(60)$ & $10(100)$ & $30(100)$ & $46(92)$ \\
\hline 72 (EgAgB1 subunit) & $6(54.5)$ & $10(83.3)$ & $12(100)$ & $10(76.9)$ & $7(63.6)$ & $45(76.3)$ & $7(70)$ & $10(100)$ & $30(100)$ & $47(94)$ \\
\hline 73 (EgAgB1/1) & $5(45.6)$ & $11(91.7)$ & $12(100)$ & $4(30.8)$ & $0(0)$ & $32(54.2)$ & $5(50)$ & $8(80)$ & $29(96.7)$ & $42(84)$ \\
\hline 74 (EgAgB1/1) & $4(36.4)$ & $10(83.3)$ & $12(100)$ & $5(38.5)$ & $4(36.4)$ & $32(54.2)$ & $7(70)$ & $10(100)$ & $30(100)$ & $47(94)$ \\
\hline 75 (EgAgB2) & $4(36.4)$ & $10(83.3)$ & $12(100)$ & $5(38.5)$ & $4(36.4)$ & $35(59.3)$ & $5(50)$ & $7(70)$ & $30(100)$ & $42(84)$ \\
\hline $77(\mathrm{EgAgB} 1 / 1)$ & $4(36.4)$ & $12(100)$ & $12(100)$ & $5(38.5)$ & $6(54.5)$ & $39(66.1)$ & $6(60)$ & $10(100)$ & $30(100)$ & $46(92)$ \\
\hline
\end{tabular}

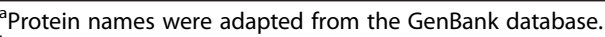

${ }^{b}$ Others included sparganosis, primary hepatocellular carcinoma, and normal controls (each of $n=10$ ).

might be readily accessible to the immune system [12,22], although its specificity is fairly low. EgAg5 is a highmolecular-weight glycoprotein complex $(>500 \mathrm{kDa})$ consisting of 57 and $67 \mathrm{kDa}$ under reducing conditions, and is dissociated into 22 and $38 \mathrm{kDa}$ subunits [22]. The protein has been widely used for CE immunodiagnosis, while other studies have demonstrated that EgAg5 cross-reacted with the patient sera of other helminthic infections [12,23]. Indeed, several EgAg5 molecules showed cross-reactions with $\mathrm{AE}$ and neurocysticercosis sera (Figure 4 and Table 2 ). In the early stage of CE, the immunoreactivity of $\mathrm{EgAgB}$ isoforms was reasonably weak. Previous studies involving animal CE immunodiagnosis revealed that EgAgB demonstrated quite low sensitivity $[24,25]$. This result strongly suggested that these animals, even infected, might be in an early stage of CE since the cattle slaughtered in abattoirs are mostly young. Sera from these animals might not respond to EgAgB as shown in this study. These collective results indicated that EgAgB proteoforms may not be applicable for serodiagnosis of early and chronic stages of CE.

As cyst(s) develops and progresses into the active stage, a substantially expanded germinal layer synthesizes and secretes copious quantities of several tens of
EgAgB, and induces antibody responses. In the transitional CE3 period, previously secreted diverse EgAgB molecules continuously stimulate the immune system. A partially degenerative germinal layer might maintain its capacity to produce and secret EgAgBs, which results in the strong antibody responses, as is seen in this study. Among several EgAgB proteoforms, EgAgB1 constituted the majority with the strongest antigenicity. When metacestode(s) regressed to chronic inactive stages, EgAgBenriched HF might be rapidly drained and become absorbed through degenerative membranes. Subsequently, the synthesis of EgAgB might also be attenuated and the antibody responses culminating to EgAgB would become negligible. A similar phenomenon has been observed in neurocysticercosis, which is caused by a phylogentically-close neighbor, the Taenia solium metacestode. Expression of a 150-kDa hydrophobic-ligandbinding-protein (HLBP) complex, which invokes specific and strong antibody reactions during the active stage, is drastically down-regulated as the parasite undergoes degeneration by calcification [26,27]. This common pattern of expressional regulation of HLBPs may have significant pathophysiological implications in the long-term survival of larval taeniid, such as E. granulosus and T. solium 
metacestodes within host environments, since HLBPs are critical in recruiting essential host lipids, regulating local immune responses, and modulating protein turnover $[17,26,27]$. This result also raised an intriguing suggestion that blocking the synthesis or inactivating these molecules might be an attractive target to control $\mathrm{CE}$ and neurocysticercosis. Molecular mechanisms underlining expressional regulation of several EgAgB proteoforms should form the basis of future studies.

The EgAgB2 appeared to be a reliable antigenic protein for CE serodiagnosis, when the HFs of bovine and human origin were employed as antigens [28]. However, such a potent immunoreactivity of EgAgB2 proteoforms was not observed with ovine CE2 HF in this study. It would be interesting to analyze whether the expressions of different EgAgBs are differentially regulated in different hosts. We could not detect individual EgAgB3-related molecules through 2-DE analysis. These molecules were only identifiable by LC-ESI-MS/MS. Since EgAgB3 is primarily synthesized in the protoscolex [29], our result suggests that, although transcription levels of EgAgB3 are relatively high in the protoscolex [14], secretion of EgAgB3 at protein levels would be minimal, and correlated with the finding that its antigenicity was reasonably weak and was detected only in the active and transitional periods, during which active immune systems are operating (Figure 3). In addition, we were unable to detect EgAgB5 molecules, which harbored similar primary sequences to EgAgB3 molecules. This isoform was indeed shown to be mainly expressed in the immature adult, but not the metacestode, stage [14,21].

CE cyst thrives for over 20 years in immunologically competent hosts. The parasite continuously interacts with the host to defend the cytopathic environment. In addition to diverse EgAgB, HF contains many secretory proteins, which may contribute to parasite survival through the regulation of bioactive molecules [29,30]. Some metabolic enzymes, especially those involved in carbohydrate metabolism, are known to perform several moonlighting activities [31-33]. We identified multifaceted E. granulosus protein enzymes, such as phosphoenolpyruvate carboxykinase (maintenance of cytosolic and mitochondrial redox balance, and induction of T-cell immunity), citrate synthase (chaperon activity, growth, and virulence), fructose 1,6 bisphosphate aldolase (cellular adhesion and antioxidant activity), and triosephosphate isomerase (anti-oxidative processes and glutathione redox cycle) [33-36]. In this study, we were able to detect host-derived serum components, antioxidant/detoxification/xenobiotic proteins, enzymes involved in carbohydrate and amino acid metabolism, and fatty-acid-binding proteins, which might be absorbed through transporter-mediated-uptake or endocytosis [26,37]. These proteins might exert their moonlighting effects within the HF. Dynamic molecular biochemical and cellular events might be actively occurring within the HF, and Echinococcus might absorb and exploit these molecules to maintain its homeostatic function [36]. Further research is warranted to explicate the biological roles of individual proteins together with the molecular network and cross-talk functions within the HF.

\section{Conclusions}

Early detection of CE may have a great impact on reduction of disability-adjusted life years, because the highest morbidity is observed in young patients under 20 years old $[1,5]$. Our results convincingly demonstrate that the detection of a single defined molecule may not properly diagnose $C E$ since specific immunopotent antigens are altered according to CE progression. Serological crossreactions between $\mathrm{CE}$ and $\mathrm{AE}$ may be inherent to some extent. Surveillance of immunoproteome characteristics combined with imaging scans may be essential to differentiate $\mathrm{CE}$ from $\mathrm{AE}$ and to clarify $\mathrm{CE}$ status. Our data also highlighted the possible biological functions of $\mathrm{HF}$ proteins, which might be intimately involved in the homeostatic maintenance and pathophysiological adaptation of the parasite during long-standing infections.

\section{Additional files}

Additional file 1: Demographic and clinical features of CE patients enrolled in this study.

Additional file 2: Protein identification of sheep CE1 and CE2 HF following MALDI-TOF-MS analysis.

\section{Abbreviations}

AE: Alveolar echinococcosis; CBB: Coomassie brilliant blue G-250; CE: Cystic echinococcosis; EgAgB: Echinococcus granulosus antigen B; EgAg5: Echinococcus granulosus antigen 5; HRP: Horse-radish peroxidase; HF: Hydatid fluid; HLBP: Hydrophobic-ligand-binding-protein; IPG: Immobilized pH gradient; MALDI-TOF-MS: Matrix-assisted laser desorption/ioninzation time-of-flight mass spectrometry; nano-LC-ESI-MS/MS: Nano-liquid chromatography-electrospray ionization/multi-stage MS; ECL: SuperSignal chemiluminescence; PMF: Peptide mass fingerprint; 2-DE: Two-dimensional electrophoresis; US: Ultrasonography.

\section{Competing interests}

The authors declare that they have no competing interests.

\section{Authors' contributions}

Experimental conception and design: YK; sample collection: $X H, X M, H C, H J Y$, CSA, JTK, HW; field survey: XH, XM, HC, HJY, YK, HW; 2-DE and immunoblot: CSA, YAB, JTK, IK; proteome analyses: CSA, IK, YAB, YK; paper writing: YK, CSA. All authors read and approved the final version of the manuscript.

\section{Acknowledgements}

We thank all local health workers of Qinghai Province Institute for Endemic Diseases Prevention and Control, China, who helped us during the survey of the patients. This work was supported by a grant from Samsung Biomedical Research Institute (SMX1132101).

\section{Author details}

${ }^{1}$ Department of Molecular Parasitology, Sungkyunkwan University School of Medicine and Center for Molecular Medicine, Samsung Biomedical Research Institute, Suwon 440-746, Korea. ${ }^{2}$ Qinghai Province Institute for Endemic Diseases Prevention and Control, Xining, Qinghai, China. ${ }^{3}$ Department of Microbiology, Graduate School of Medicine, Gachon University, Incheon, 
Korea. ${ }^{4}$ Department of Parasitology, Ewha Womans University, School of Medicine, Seoul, Korea. ${ }^{5}$ Department of Molecular Biology and Biochemistry, School of Medicine, Kyung Hee University, Seoul, Korea.

Received: 30 September 2014 Accepted: 17 December 2014 Published online: 08 January 2015

\section{References}

1. Eckert J, Deplazes P: Biological, epidemiological, and clinical aspects of echinococcosis, a zoonosis of increasing concern. Clin Microbiol Rev 2009, 27:107-135.

2. Zhang W, Zhang Z, Wu W, Shi B, Li J, Zhou X, Wen H, McManus DP: Epidemiology and control of echinococcosis in central Asia, with particular reference to the People's Republic of China. Acta Trop 2014. http://dx.doi.org/10.1016/j.actatropica.2014.03.014.

3. Yang YR, Clements AC, Gray DJ, Atkinson JA, Williams GM, Barnes TS, McManus DP: Impact of anthropogenic and natural environmental changes on Echinococcus transmission in Ningxia Hui Autonomous Region, the People's Republic of China. Parasit Vectors 2012, 5:146.

4. Craig PS, McManus DP, Lightowlers MW, Chabalgoity JA, Garcia HH, Gavidia CM, Gilman RH, Gonzalez AE, Lorca M, Naquira C, Nieto A, Schantz PM: Prevention and control of cystic echinococcosis. Lancet Infect Dis 2007, 7:385-394.

5. Brunetti E, Kern P, Vuitton DA: Writing panel for the WHO-IWGE. expert consensus for the diagnosis and treatment of cystic and alveolar echinococcosis in humans. Acta Trop 2010, 114:1-16.

6. Caremani M, Maestrini R, Occhini U, Sassoli S, Accorsi A, Giorgio A, Filice C: Echographic epidemiology of cystic hydatid disease in Italy. Eur J Epidemiol 1993, 9:401-404.

7. Perdomo R, Alvarez C, Monti J, Ferreira C, Chiesa A, Carbó A, Alvez R, Grauert R, Stern D, Carmona C, Yarzabal L: Principles of the surgical approach in human liver cystic echinococcosis. Acta Trop 1997, 64:109-122.

8. Eckert J, Gemmell MA, Meslin FX, Pawlowski ZS: WHO/OIE Manual on Echinococcosis in Humans and Animals: a Public Health Problem of Global Concern. Paris: WHO/OIE; 2001.

9. Taheri MS, Pourghorban R, Faghihi Langroudi T, Shabestari AA, Haghighatkhah H, Bagheri SM: Not-to-tap cystic lesions: spectrum of hydatid disease. Emerg Radiol 2013, 20:553-561.

10. Siracusano A, Margutti P, Delunardo F, Profumo E, Riganò R, Buttari B, Teggi A, Ortona E: Molecular cross-talk in host-parasite relationships: the intriguing immunomodulatory role of Echinococcus antigen B in cystic echinococcosis. Int J Parasitol 2008, 38:1371-1376.

11. Ortona E, Riganò R, Margutti P, Notargiacomo S, loppolo S, Vaccari S, Barca S, Buttari B, Profumo E, Teggi A, Siracusano A: Native and recombinant antigens in the immunodiagnosis of human cystic echinococcosis. Parasite Immunol 2000, 22:553-559.

12. Lorenzo C, Last JA, González-Sapienza GG: The immunogenicity of Echinococcus granulosus antigen 5 is determined by its post-translational modifications. Parasitology 2005, 131:669-677.

13. Haag KL, Alves-Junior L, Zaha A, Ayala FJ: Contingent, non-neutral evolution in a multicellular parasite: natural selection and gene conversion in the Echinococcus granulosus antigen B gene family. Gene 2004, 333:157-167.

14. Zhang W, Li J, Jones MK, Zhang Z, Zhao L, Blair D, McManus DP: The Echinococcus granulosus antigen $\mathrm{B}$ gene family comprises at least 10 unique genes in five subclasses which are differentially expressed. PLoS Negl Trop Dis 2010, 4:e784.

15. Shepherd JC, Aitken A, McManus DP: A protein secreted in vivo by Echinococcus granulosus inhibits elastase activity and neutrophil chemotaxis. Mol Biochem Parasitol 1991, 44:81-90.

16. Daeki AO, Craig PS, Shambesh MK: IgG-subclass antibody responses and the natural history of hepatic cystic echinococcosis in asymptomatic patients. Ann Trop Med Parasitol 2000, 94:319-328.

17. Obal G, Ramos AL, Silva V, Lima A, Batthyany C, Bessio MI, Ferreira F, Salinas G, Ferreira AM: Characterisation of the native lipid moiety of Echinococcus granulosus antigen B. PLoS Negl Trop Dis 2012, 6:e1642

18. Junghanss T, da Silva AM, Horton J, Chiodini PL, Brunetti E: Clinical management of cystic echinococcosis: state of the art, problems, and perspectives. Am J Trop Med Hyg 2008, 79:301-311.

19. Mariconti M, Bazzocchi C, Tamarozzi F, Meroni V, Genco F, Maserati R, Brunetti E: Immunoblotting with human native antigen shows stage- related sensitivity in the serodiagnosis of hepatic cystic echinococcosis. Am J Trop Med Hyg 2014, 90:75-79.

20. Bae YA, Jeong YT, Chung JY, Kim SH, Mahanta J, Feng Z, Chong CK, Kim TS, Kong Y: A recombinant chimeric antigen toward a standardized serodiagnosis of Taenia solium neurocysticercosis. Proteomics Clin Appl 2008, 2:1596-1610

21. Monteiro KM, Cardoso MB, Follmer C, da Silveira NP, Vargas DM, Kitajima EW, Zaha A, Ferreira HB: Echinococcus granulosus antigen B structure: subunit composition and oligomeric states. PLoS Negl Trop Dis 2012, 6:e1551.

22. Lightowlers MW, Liu DY, Haralambous A, Rickard MD: Subunit composition and specificity of the major cyst fluid antigens of Echinococcus granulosus. Mol Biochem Parasitol 1989, 37:171-182.

23. Khabiri AR, Bagheri F, Assmar M, Siavashi MR: Analysis of specific lgE and IgG subclass antibodies for diagnosis of Echinococcus granulosus. Parasite Immunol 2006, 28:357-362.

24. Ibrahem MM, Rafiei A, Dar FK, Azwai SM, Carter SD, Craig PS: Serodiagnosis of cystic echinococcosis in naturally infected camels. Parasitology 2002, 125:245-251.

25. McManus DP: Immunodiagnosis of sheep infections with Echinococcus granulosus: in 35 years where have we come? Parasite Immunol 2004, 36:125-130.

26. Lee EG, Kim SH, Bae YA, Chung JY, Suh M, Na BK, Kim TS, Kang I, Ma L, Kong Y: A hydrophobic ligand binding protein of the Taenia solium metacestode mediates uptake of the host lipid: implication for the maintenance of parasitic cellular homeostasis. Proteomics 2007, 7:4016-4030

27. Kim SH, Bae YA, Yang Y, Hong ST, Kong Y: Paralogous proteins comprising the $150 \mathrm{kDa}$ hydrophobic-ligand-binding-protein complex of the Taenia solium metacestode have evolved non-overlapped binding affinities toward fatty acid analogs. Int J Parasitol 2011, 41:1207-1215.

28. Virginio VG, Hernández A, Rott MB, Monteiro KM, Zandonai AF, Nieto A, Zaha A, Ferreira HB: A set of recombinant antigens from Echinococcus granulosus with potential for use in the immunodiagnosis of human cystic hydatid disease. Clin Exp Immunol 2003, 132:309-315.

29. Virginio VG, Monteiro KM, Drumond F, de Carvalho MO, Vargas DM, Zaha A, Ferreira HB: Excretory/secretory products from in vitro-cultured Echinococcus granulosus protoscoleces. Mol Biochem Parasitol 2012, 183:15-22.

30. Cui SJ, Xu LL, Zhang T, Xu M, Yao J, Fang CY, Feng Z, Yang PY, Hu W, Liu F: Proteomic characterization of larval and adult developmental stages in Echinococcus granulosus reveals novel insight into host-parasite interactions. J Proteomics 2013, 84:158-175.

31. Gancedo C, Flores C: Moonlighting proteins in yeasts. Microbiol Mol Biol Rev 2008, 72:197-210.

32. Pomel S, Luk FC, Beckers CJ: Host cell egress and invasion induce marked relocations of glycolytic enzymes in Toxoplasma gondii tachyzoites. PLOS Pathog 2008, 4:e1000188.

33. Lorenzatto KR, Monteiro KM, Paredes R, Paludo GP, da Fonsêca MM, Galanti N, Zaha A, Ferreira HB: Fructose-bisphosphate aldolase and enolase from Echinococcus granulosus: genes, expression patterns and protein interactions of two potential moonlighting proteins. Gene 2012, 506:76-84.

34. Buchner J, Grallert H, Jakob U: Analysis of chaperone function using citrate synthase as nonnative substrate protein. Methods Enzymol 1998, 290:323-338.

35. Asahi H, Osman A, Cook RM, LoVerde PT, Stadecker MJ: Schistosoma mansoni phosphoenolpyruvate carboxykinase, a novel egg antigen: immunological properties of the recombinant protein and identification of a T-cell epitope. Infect Immun 2000, 68:3385-3393.

36. Monteiro KM, de Carvalho MO, Zaha A, Ferreira HB: Proteomic analysis of the Echinococcus granulosus metacestode during infection of its intermediate host. Proteomics 2010, 10:1985-1999.

37. Ambrosio J, Landa A, Merchant MT, Laclette JP: Protein uptake by cysticerci of Taenia crassiceps. Arch Med Res 1994, 25:325-330. 